\title{
Focal unspecific bone uptake on [ 18 F]-PSMA-1007 PET: a multicenter retrospective evaluation of the distribution, frequency, and quantitative parameters of a potential pitfall in prostate cancer imaging
}

Grünig, Hannes ; Maurer, Alexander ; Thali, Yannick ; Kovacs, Zsofia ; Strobel, Klaus ; Burger, Irene A ; Müller, Joachim

\begin{abstract}
Purpose: Improved logistics and availability led to a rapid increase in the use of [18F]-PSMA1007 for prostate cancer PET imaging. Initial data suggests increased uptake in benign lesions compared to [68 Ga]-PSMA-11, and clinical observations found increased unspecific bone uptake (UBU). We therefore investigate the frequency and characteristics of UBU in [18F]-PSMA-1007 PET. Methods: We retrospectively analyzed [18F]-PSMA-1007 PET scans from four centers for the presence of UBU, defined as a focal mild-to-moderate uptake (SUVmax < 10.0) not obviously related to a benign or malignant cause. If present, up to three leading UBUs were quantified (SUVmax), localized, and correlated to clinical parameters, such as age, PSA, injected dose, Gleason score, tumor size (T1-T4), and type of PET scanner (analog vs. digital). Additionally, clinical and imaging follow-up results and therapeutic impact were evaluated. Results: UBUs were identified in 179 out of 348 patients $(51.4 \%)$. The most frequent localizations were ribs (57.5\%) and pelvis (24.8\%). The frequency of UBUs was not associated with PSA, Gleason score, tumor size, age, or the injected [18F]-PSMA-1007 dose. UBUs were significantly more frequent in images obtained with digital PET/CT scans $(\mathrm{n}=74,82 \%)$ than analog PET/CT scans $(\mathrm{n}=$ $221,40.3 \%)(\mathrm{p}=.0001)$ but not in digital PET/MR $(\mathrm{n}=53,51 \%)(\mathrm{p}=.1599)$. In 80 out of 179 patients $(44.7 \%)$, the interpretation of UBUs was critical for therapeutic management and therefore considered clinically relevant. For 65 UBUs, follow-ups were available: three biopsies, three radiotherapies with PSA follow-up, and 59 cases with imaging. After follow-up, UBUs were still considered unclear in 28 of 65 patients $(43 \%)$, benign in $28(43 \%)$, and malignant in nine (14\%) patients. Conclusion: UBUs occur in two-thirds of patients imaged with [18F]-PSMA-1007 PET/CT and are significantly more frequent on digital PET scanners than analog scanners. UBUs should be interpreted carefully to avoid over-staging. Keywords: Bone metastasis; PET/CT; PET/MR; Restaging; Staging; [18F]-PSMA.
\end{abstract}

DOI: https://doi.org/10.1007/s00259-021-05424-x

Posted at the Zurich Open Repository and Archive, University of Zurich

ZORA URL: https://doi.org/10.5167/uzh-208839

Journal Article

Published Version

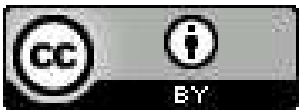

The following work is licensed under a Creative Commons: Attribution 4.0 International (CC BY 4.0) License. 
Originally published at:

Grünig, Hannes; Maurer, Alexander; Thali, Yannick; Kovacs, Zsofia; Strobel, Klaus; Burger, Irene A; Müller, Joachim (2021). Focal unspecific bone uptake on [ 18 F]-PSMA-1007 PET: a multicenter retrospective evaluation of the distribution, frequency, and quantitative parameters of a potential pitfall in prostate cancer imaging. European Journal of Nuclear Medicine and Molecular Imaging, 48(13):44834494.

DOI: https://doi.org/10.1007/s00259-021-05424-x 


\title{
Focal unspecific bone uptake on $\left[{ }^{18} \mathrm{~F}\right]-\mathrm{PSMA}-1007$ PET: a multicenter retrospective evaluation of the distribution, frequency, and quantitative parameters of a potential pitfall in prostate cancer imaging
}

\author{
Hannes Grünig ${ }^{1}(\mathbb{D}) \cdot$ Alexander Maurer $^{1}(\mathbb{D}) \cdot$ Yannick Thali $^{2} \cdot$ Zsofia Kovacs $^{3} \cdot$ Klaus Strobel $^{2}$ (D) Irene A. Burger $^{1,4}(\mathbb{D} \cdot$ \\ Joachim Müller ${ }^{3}$ (D)
}

Received: 17 March 2021 / Accepted: 19 May 2021 / Published online: 13 June 2021

(C) The Author(s) 2021

\begin{abstract}
Purpose Improved logistics and availability led to a rapid increase in the use of $\left[{ }^{18} \mathrm{~F}\right]$-PSMA-1007 for prostate cancer PET imaging. Initial data suggests increased uptake in benign lesions compared to $\left[{ }^{68} \mathrm{Ga}\right]$-PSMA-11, and clinical observations found increased unspecific bone uptake (UBU). We therefore investigate the frequency and characteristics of UBU in $\left[{ }^{18}\right.$ F]-PSMA-1007 PET.

Methods We retrospectively analyzed $\left[{ }^{18} \mathrm{~F}\right]$-PSMA-1007 PET scans from four centers for the presence of UBU, defined as a focal mild-to-moderate uptake $\left(\mathrm{SUV}_{\max }<10.0\right)$ not obviously related to a benign or malignant cause. If present, up to three leading UBUs were quantified $\left(\mathrm{SUV}_{\max }\right)$, localized, and correlated to clinical parameters, such as age, PSA, injected dose, Gleason score, tumor size (T1-T4), and type of PET scanner (analog vs. digital). Additionally, clinical and imaging follow-up results and therapeutic impact were evaluated.

Results UBUs were identified in 179 out of 348 patients (51.4\%). The most frequent localizations were ribs (57.5\%) and pelvis (24.8\%). The frequency of UBUs was not associated with PSA, Gleason score, tumor size, age, or the injected $\left[{ }^{18} \mathrm{~F}\right]$-PSMA-1007 dose. UBUs were significantly more frequent in images obtained with digital PET/CT scans $(\mathrm{n}=74$, $82 \%)$ than analog PET/CT scans $(\mathrm{n}=221,40.3 \%)(p=.0001)$ but not in digital PET/MR $(\mathrm{n}=53,51 \%)(p=.1599)$. In 80 out of 179 patients $(44.7 \%)$, the interpretation of UBUs was critical for therapeutic management and therefore considered clinically relevant. For 65 UBUs, follow-ups were available: three biopsies, three radiotherapies with PSA follow-up, and 59 cases with imaging. After follow-up, UBUs were still considered unclear in 28 of 65 patients (43\%), benign in 28 (43\%), and malignant in nine (14\%) patients.

Conclusion UBUs occur in two-thirds of patients imaged with $\left[{ }^{18} \mathrm{~F}\right]-\mathrm{PSMA}-1007$ PET/CT and are significantly more frequent on digital PET scanners than analog scanners. UBUs should be interpreted carefully to avoid over-staging.
\end{abstract}

Keywords Staging $\cdot$ Restaging $\cdot\left[{ }^{18} \mathrm{~F}\right]$-PSMA $\cdot$ Bone metastasis $\cdot \mathrm{PET} / \mathrm{CT} \cdot \mathrm{PET} / \mathrm{MR}$

This article is part of the Topical Collection on Oncology-

Genitourinary

Hannes Grünig and Alexander Maurer shared the first authorship Irene A. Burger and Joachim Müller shared the last authorship

Irene A. Burger

irene.burger@ksb.ch

1 Department of Nuclear Medicine, University Hospital Zurich, University of Zurich, Zurich, Switzerland

2 Department of Nuclear Medicine and Radiology, Cantonal Hospital Lucerne, Lucerne, Switzerland
3 Department of Radiology and Nuclear Medicine, Cantonal Hospital St. Gallen, St. Gallen, Switzerland

4 Department of Nuclear Medicine, Cantonal Hospital Baden, Baden, Switzerland 


\section{Introduction}

Positron emission tomography (PET), combined with either computer tomography (CT) or magnetic resonance imaging (MRI), utilizing radiotracers that bind to prostate-specific membrane antigen (PSMA) is an excellent diagnostic tool for prostate cancer imaging. In the past few years, PSMA-PET has evolved to become the leading advanced imaging modality, especially for patients with early biochemical recurrence (BCR) [1,2]. In various studies, PSMA-PET/CT has shown better detection efficacy in early BCR than MRI, CT, conventional imaging [3-6], or choline-labeled PET ligands [7], and showed substantial impact on management [8]. Moreover, the examination is gaining increasing importance for the initial staging of intermediate and high-risk prostate cancer [9-14].

Several PSMA ligands are available, radiolabeled with currently primary two different positron-emitting isotopes: gallium-68 [ $\left.{ }^{68} \mathrm{Ga}\right]$ and fluorine-18 $\left[{ }^{18} \mathrm{~F}\right]$.The most commonly used PSMA agent in Europe was initially [ $\left.{ }^{68} \mathrm{Ga}\right]-$ PSMA-11, with well-established application for cancer localization in early BCR, with high detection rates, and an impact on management following $60 \%$ of scans [15]. In the USA, $\left[{ }^{68} \mathrm{Ga}\right]$-PSMA-11 was the first prostate cancer PET tracer to be approved in December 2020 [16]. More recently, $\left[{ }^{18} \mathrm{~F}\right]$-PSMA ligands have become more available, replacing $\left[{ }^{68} \mathrm{Ga}\right]$-PSMA ligands. The $\left[{ }^{18} \mathrm{~F}\right]$-labeled DCFPyL showed promising results with high image quality and good lesion detection [17], and similar results were also observed with a new class of radiohybrid tracers (rhPSMA-7) that can be labeled with $\left[{ }^{18} \mathrm{~F}\right]$ or metals such as ${ }^{68} \mathrm{Ga}$ ], respectively [18]. The major technical advantages of $\left[{ }^{18} \mathrm{~F}\right]$-PSMA ligands over $\left[{ }^{68} \mathrm{Ga}\right]$-PSMA ligands are the longer half-life (110 min vs. $68 \mathrm{~min})$ and higher production capacity, as it is produced in cyclotrons rather than generators, resulting in greater availability and fewer logistical challenges. The lower positron emission energy of $\left[{ }^{18} \mathrm{~F}\right]$-PSMA ligands than $\left[{ }^{68} \mathrm{Ga}\right]$-PSMA ligands $(0.6 \mathrm{MeV}$ vs. $2.3 \mathrm{MeV})$ also leads to a higher image resolution in comparative phantom studies [19]. One of the candidate ligands already implemented in clinical routines in several hospitals in Switzerland is $\left[{ }^{18} \mathrm{~F}\right]$-PSMA-1007, which benefits from low background activity in the urinary tract [20], which is an important advantage in suspected local recurrence $[5,21]$.

However, with the greater use of $\left[{ }^{18} \mathrm{~F}\right]-\mathrm{PSMA}-1007$, initial studies have reported a higher frequency of unclear focal uptakes than for $\left[{ }^{68} \mathrm{Ga}\right.$ ]-PSMA ligands, especially in the lymph nodes, ganglia, and bones [22]. In contrast to the nonspecific uptake in the axillary or mediastinal lymph nodes and the physiologic uptake in the ganglia, unspecific bone uptake (UBU) without morphological correlates might be interpreted as metastasis, with the potential for over-staging the patient, leading to inadequate therapy. The aim of this retrospective multicenter study was to analyze the frequency, anatomical distribution, characteristics, and influencing parameters for UBUs in $\left[{ }^{18} \mathrm{~F}\right]$-PSMA-1007 PET and to evaluate their therapeutic impact.

\section{Methods}

\section{Study design and population}

In this study, we analyzed all $\left[{ }^{18} \mathrm{~F}\right]-\mathrm{PSMA}-1007$ scans from four centers (centers A, B, C, and D) obtained between October 2019 and July 2020. This retrospective multicenter study was approved by the lead ethics committee, with general consent present at three centers and waived in one center (EKNZ ID: 2020-01,775). Only patients rejecting the general consent were excluded from the study, which was conducted in compliance with ICH-GCP rules and the Declaration of Helsinki.

Patients with histology-proven prostate cancer of any tumor stage underwent $\left[{ }^{18} \mathrm{~F}\right]-\mathrm{PSMA}-1007-\mathrm{PET}$ due to early BCR, for staging, or for general tumor evaluation (TE). Patient characteristics were collected, including age, initial tumor stage (TNM classification) if available, ISUP score for histological grading [23], bone metastasis, and PSA value less than 4 weeks before the scan. For patients with repeat $\left[{ }^{18} \mathrm{~F}\right]$-PSMA-1007-PET during the study period, only the first scan was included and further imaging used for follow-up analysis.

A hybrid PET/CT scanner or a hybrid PET/MR scanner incorporating MR and PET scanners with time of flight was used for the acquisition of the datasets. Imaging was performed using five analog PET/CT scanners (GE PETCT Discovery 600 and 690, GE Healthcare, Waukesha, WI; Siemens PET/CT Biograph mCT Flow, Siemens Healthineers, Munich, Germany), two digital PET/CT scanners (GE Discovery Molecular Insights - DMI PET/CT, GE Healthcare, Waukesha, WI), and one digital PET/MR with silicon photomultiplier technology (Signa PET/MR, GE Healthcare, Waukesha, WI, USA). At center A, only digital PET scanners were available (two PET/CT and one PET/ MR). The injected dose of $\left[{ }^{18} \mathrm{~F}\right]-\mathrm{PSMA}$ was $3-4 \mathrm{MBq} / \mathrm{kg}$ at all the centers, and the uptake time was $60-90 \mathrm{~min}$. The maximal injected dose was not more than $350 \mathrm{MBq}$. Imaging protocols in detail for all four centers are presented in Supp. 1. The study flow diagram is presented in Fig. 1.

\section{UBU-based assessment}

All scans were analyzed by one reader with access to clinical information for the presence of UBU, defined as lesions 
Fig. 1 Study flow diagram. RT: radiotherapy

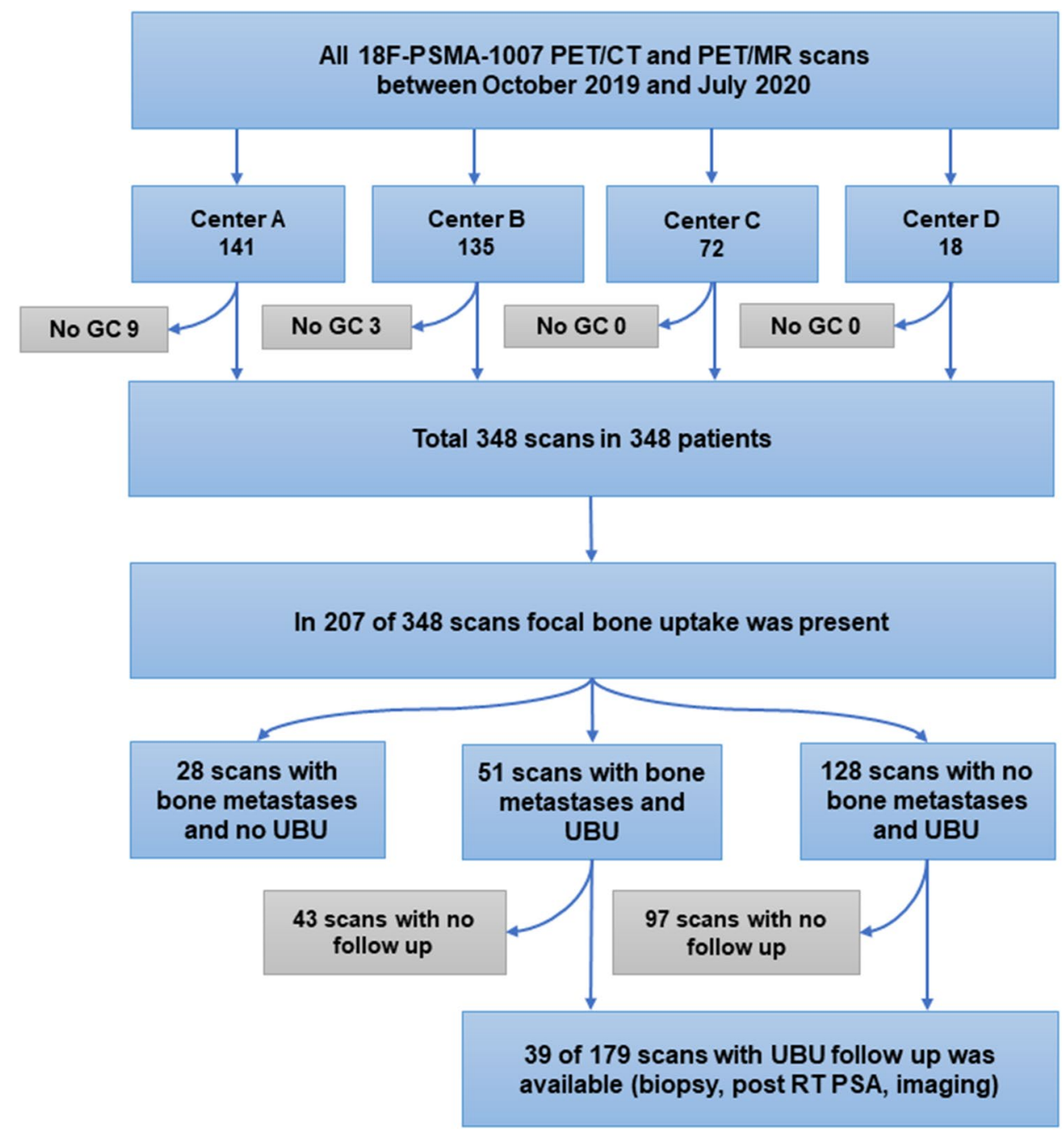

with $\mathrm{SUV}_{\max }$ below 10 and with neither morphological correlates that suggest metastatic disease nor clear benign findings, such as inflammatory joint diseases, fibrous dysplasia, or fractures. Based on previous observations, bone UBUs have an uptake between $\mathrm{SUV}_{\max } 3$ and 10 [24]. If present, the three most active UBUs in each patient were quantified $\left(\mathrm{SUV}_{\max }\right)$ and localized (Fig. 2). UBUs were localized in the skull, spine, ribs, sternum, pelvis, and extremities. The datasets were read by physicians who were double boardcertified in radiology and nuclear medicine.

\section{Patient-based assessment}

The relationships between the frequency of UBUs and clinical parameters such as age (years), PSA (ng/ml), PSMA uptake time, Gleason score categorized according to the International Society of Urological Pathology (ISUP), with prognostic grade groups 1 to 5 , and tumor size (T1 to T4) were analyzed. For patients with UBUs, the therapeutic impact was evaluated: A clinical problem $(\mathrm{CP})$ was considered to be present if the UBUs would alter management when judged as probably benign or malignant-for example, in patients with early BCR with a solitary UBU in the skeleton or in patients referred for staging with UBUs that would prevent local radical therapy if interpreted as malignant. No CP due to UBUs was considered present with either multiple bone metastasis or several clear lesions that had already been excluded from targeted therapy. Furthermore, we retrospectively analyzed whether further clinical investigations were performed, such as additional imaging, biopsy, or PSA value, after radiotherapy. For those patients, the UBUs were classified as malignant, benign, or still unclear based on the follow-up investigation.

\section{Differences between institutions and scanners}

The numbers of patients, numbers of analog and digital PET scanners, frequency of UBUs, and mean $\mathrm{SUV}_{\max }$ of the UBUs for each center were collected. The relationships between the frequency of UBUs and both the injected dose 

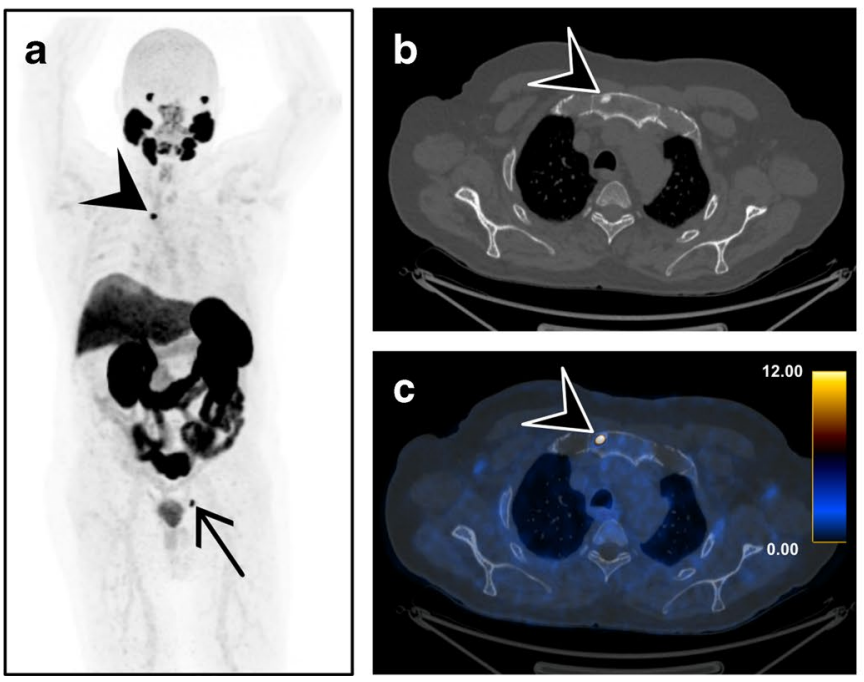

Fig. $2\left[{ }^{18} \mathrm{~F}\right]-\mathrm{PSMA}-1007-\mathrm{PET} / \mathrm{CT}$ on a digital scanner illustrates bone metastasis and UBU in two different patients with prostate cancer. a A whole-body maximum intensity projection of PET shows a high PSMA-positive bone lesion $\left(\mathrm{SUV}_{\max } 23.8\right)$ in the manubrium sterni (arrowhead) and a lymph node metastasis in the left pelvis (thin black arrow). b Focal sclerosis in the manubrium sterni (arrow-

of $\left[{ }^{18} \mathrm{~F}\right]-\mathrm{PSMA}-1007$ and scanner type (analog vs. digital) were analyzed.

\section{Statistical analysis}

Continuous variables were summarized as medians and IQR, and categorical variables were summarized as numbers and percentages. All continuous variables were tested for normal distribution with the D'Agostino-Pearson test, and normality was rejected if $p<0.05$. Continuous data were compared with the Mann-Whitney test, and the $\mathrm{U}, \mathrm{Z}$, and $p$ values and 95\% CI were calculated and presented in box-whisker plots. Categorical data were compared using chi-squared tests; if significant, Pearson's contingency coefficient (C) was calculated. Sankey diagrams were used to visualize the clinical impact of UBUs and the outcomes of different followup methods. A $p$-value $<0.05$ was considered statistically significant in all cases. Statistical analysis was performed using the MedCalc Statistical Software version 19.1 (MedCalc Software bv, Ostend, Belgium). Sankey diagrams were designed with $e$ !Sankey 5.2.1 (ifu Institut für Umweltinformatik Hamburg GmbH, Hamburg, Germany).

\section{Results}

\section{Patient characteristics and demographic data}

Patient characteristics and demographic data are shown in Table 1.
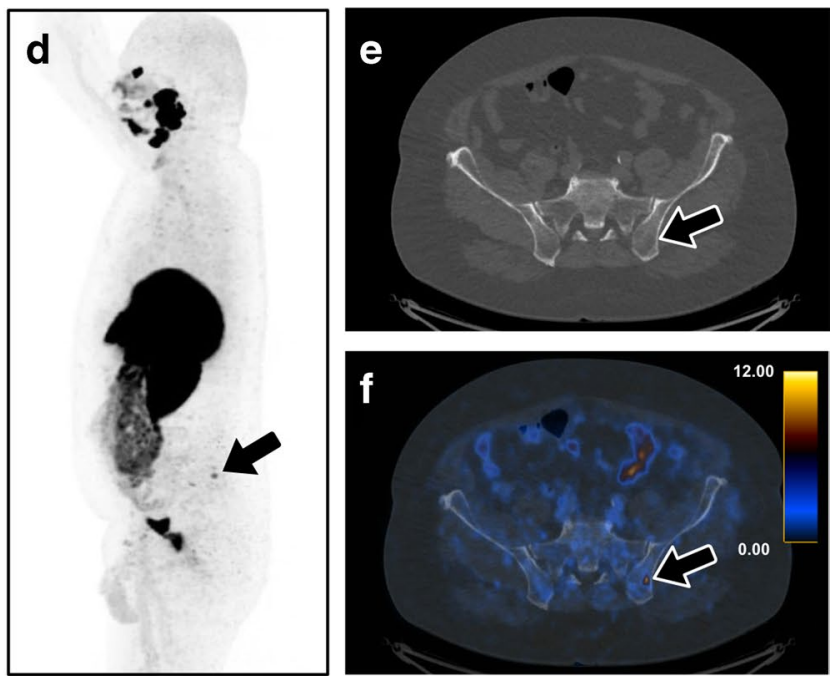

head) on axial CT, c corresponding to the high PSMA uptake on the fused images, suggests bone metastasis. d A whole-body maximum intensity projection of PET with a moderate PSMA-positive lesion $\left(\mathrm{SUV}_{\max }\right.$ 9.5) in the left iliac bone (bold arrow), e without morphological correlation on axial CT, was rated as an unspecific finding on f PET/CT imaging

\section{UBU-based assessment}

A total of 348 scans were evaluated, and 351 UBUs were selected in $179(51.4 \%)$ of the patients. Overall the median of UBUs per patient was 2 (IQR 1-4). The most frequent localization was the ribs (57.5\%), with a mean $\mathrm{SUV}_{\max }$ of 3.8 , followed by the pelvis $\left(24.8 \%, \mathrm{SUV}_{\max } 5.0\right)$, spine (9.7\%, $\left.\mathrm{SUV}_{\max } 4.7\right)$, extremities $\left(5.4 \%, \mathrm{SUV}_{\max } 4.6\right)$, sternum $\left(2.0 \%, \mathrm{SUV}_{\max } 4.3\right)$, and skull $\left(0.6 \%, \mathrm{SUV}_{\max } 5.9\right)$. The mean $\mathrm{SUV}_{\max }$ of all UBUs was $4.2 \pm 2.0$ (Fig. 3).

\section{Patient-based assessment}

Age was not associated with frequency of UBUs $(\mathrm{U}=13,014.00, \mathrm{Z}=2.25, p=0.02)$; although the $p$-value was $<0.05$, the $95 \% \mathrm{CI}$ of median difference was not significant (median difference $-2.0,95 \% \mathrm{CI}-3.0$ to 0.0 ) (Fig. 4a). The frequency of UBUs was not associated with PSA value $(\mathrm{U}=11,090.00, \mathrm{Z}=0.68, p=0.50$ ) (Fig. 4b). Chi-squared tests showed no relationship between the frequency of UBUs and either tumor size $\left(\chi^{2}(3)=5.61, p=0.0573\right)$ or ISUP score $\left(\chi^{2}(4)=4.78, p=0.3108\right)$ (Fig. $\left.4 c-d\right)$.

Overall, in 80 out of 179 patients (44.7\%) with UBUs, the lesions were considered a CP that could alter management. Regarding the indication for the PET scan, CPs were present in 55 of 227 patients with early BCR (24.2\%), in 15 of 49 for tumor staging (31\%), and in 10 of 72 for TE (14\%). UBUs with no CPs were present in 60 of 227 (26.4\%) patients with early BCR, 16 of 49 for tumor staging (33\%), and 23 of 72 for TE (32\%). Overall, 39 out of 348 patients 
Table 1 Patient characteristics and demographic data

\begin{tabular}{|c|c|}
\hline Number of patients & 348 \\
\hline Age (y) (median, IQR) & $71.0(66-76)$ \\
\hline \multicolumn{2}{|c|}{ Indication for $\left[{ }^{18} \mathrm{~F}\right]$-PSMA-1007-PET, $\mathrm{n}=348$} \\
\hline Early BCR & $227(65.2 \%)$ \\
\hline Tumor evaluation & $71(20.7 \%)$ \\
\hline Staging & $49(14.1 \%)$ \\
\hline \multicolumn{2}{|l|}{ Initial $\mathrm{T}$ classification, $\mathrm{n}=281$} \\
\hline $\mathrm{T} 1$ & $30(10.7 \%)$ \\
\hline $\mathrm{T} 2$ & $88(31.3 \%)$ \\
\hline $\mathrm{T} 3$ & $151(53.7 \%)$ \\
\hline $\mathrm{T} 4$ & $12(4.3 \%)$ \\
\hline \multicolumn{2}{|l|}{ Initial $N$ classification, $n=267$} \\
\hline No & $163(61.0 \%)$ \\
\hline N1 & $97(36.3 \%)$ \\
\hline $\mathrm{Nx}$ & $7(2.6 \%)$ \\
\hline \multicolumn{2}{|l|}{ Initial $M$ classification, $n=267$} \\
\hline M0 & $215(83.7 \%)$ \\
\hline M1 & $23(8.9 \%)$ \\
\hline $\mathrm{Mx}$ & $19(7.4 \%)$ \\
\hline \multicolumn{2}{|c|}{ Resection boundaries $(\mathrm{R}), \mathrm{n}=142$} \\
\hline $\mathrm{R} 0$ & $62(43.7 \%)$ \\
\hline $\mathrm{R} 1$ & $80(56.3 \%)$ \\
\hline Patients with bone metastasis & $79(22.7 \%)$ of 348 \\
\hline \multicolumn{2}{|c|}{ Median (IQR) of PSA values [ng/ml]; $n=306$} \\
\hline Overall & $2.5(0.5-9.3)$ \\
\hline Early BCR & $1.2(0.4-4.0)$ \\
\hline Tumor evaluation & $10.7(3.3-87.0)$ \\
\hline Staging & $11.7(11.7-34.7)$ \\
\hline \multicolumn{2}{|l|}{ ISUP grade groups, $n=291$} \\
\hline ISUP 1 & $23(7.9 \%)$ \\
\hline ISUP 2 & $48(16.5 \%)$ \\
\hline ISUP 3 & $83(28.5 \%)$ \\
\hline ISUP 4 & $74(25.4 \%)$ \\
\hline ISUP 5 & $63(21.6 \%)$ \\
\hline
\end{tabular}

Values are given as absolute numbers and percentages in parentheses or median

$B C R$ biochemical recurrence; $I Q R$ interquartile range

(13.8\%) were followed by imaging, biopsy, or radiotherapy with PSA follow-up. Only 25 out of 80 patients (31\%) with a CP were followed (Fig. 5).

Follow-up was available for 65 UBUs: three (5\%) biopsies (all benign), three (5\%) radiotherapies with PSA follow-up (two malignant and one benign) (Table 2), and 59 (91\%) imaging follow-ups (seven malignant, 24 benign, 28 unknown). Overall, 59 UBUs were followed up by imaging: 23 (39\%) by PET, 17 (29\%) by CT, 14 (24\%) by MRI, and five $(9 \%)$ by SPECT. However, after follow-up, 28 (43\%) UBUs were still considered unknown, with 28 (43\%) benign and nine (14\%) malignant (Fig. 6, table provided in Supp. 2). On a scan base, follow-up was available in 39 scans of

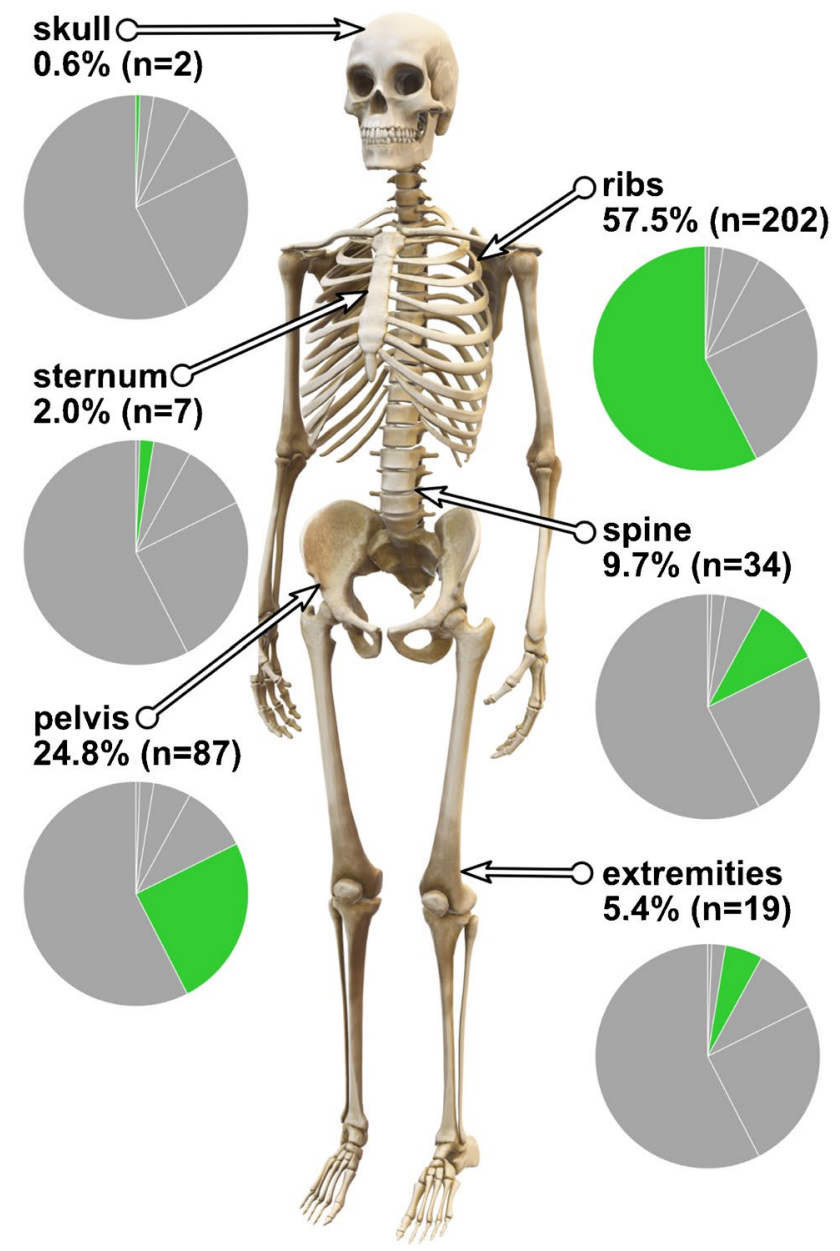

Fig. 3 Percentage and number of the three most active UBUs by anatomic region in $\left[{ }^{18} \mathrm{~F}\right]$-PSMA-1007-PET

$179(21 \%)$ with UBUs, and in $41 \%$ of the scans, all lesions were considered benign bases on follow-up data (Supp. 3).

\section{Differences between institutions and scanners}

Of the 348 PET scans, 132 (37.9\%) were performed in center A, $126(36.2 \%)$ in center B, $72(20.7 \%)$ in C, and $18(5.2 \%)$ in D; $221(63.5 \%)$ patients were scanned on an analog PET/ CT scanner, 74 (21.3\%) on a digital PET/CT scanner, and $53(15.2 \%)$ on a digital PET/MRI scanner. Only in center A were digital PET/CT scanners or PET/MRI scanners available; in center A, only six PET/CT scans were performed on an analog scanner. There were significantly more UBUs present on digital PET scanners (70.1\%) than analog scanners $(40.7 \%)\left(\chi^{2}(1)=27.74, p=0.0001, \mathrm{C}=0.27\right)$. Subanalysis shows also a higher incidence of UBUs in digital PET/CT compared with analog PET/CT $\left(\chi^{2}(1)=14.64, p=0.0001\right)$ (Fig. 7a). However, comparison of analog PET/CT with digital PET/MRI shows no difference in the incidence of UBUs $\left(\chi^{2}(1)=1.98, p=0.1599\right)$ (Fig. 7a). Uptake time of 

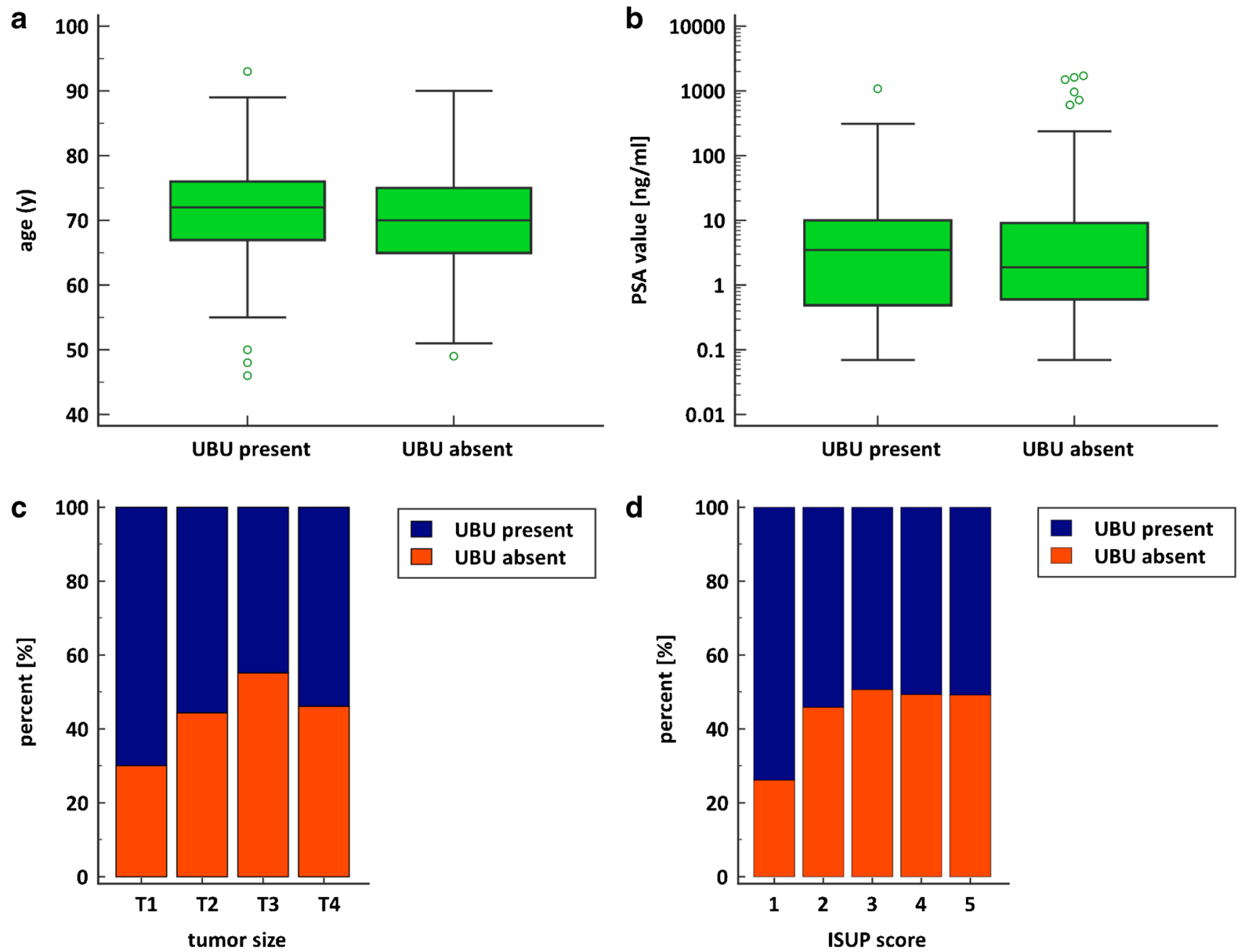

Fig. 4 a Box-whisker plots show no significant association between age and frequency of UBUs ( $p=.02$, but the $95 \%$ CI of median difference was not significant). b Box-whisker plots show no significant association between PSMA value and the frequency of UBUs

90 min was associated with a higher incidence of UBU as well $\left(\chi^{2}(1)=31.24, p<0.0001\right)$ (Fig. 7b).

The number of patients with UBUs at center A was 92 (69.7\%), at center B was 50 (39.7\%), at center C was 26 (36\%), and at center D was 11 (61\%) (Supp. 4). The mean $\mathrm{SUV}_{\max }$ and number of analyzed UBUs at center A were 4.6 and 193 (55.0\%), respectively; at center B, they were 3.9 and 98 (27.9\%), respectively; at center C, they were 3.0 and $42(12 \%)$, respectively; and at center $\mathrm{D}$, they were 5.8 and $18(5 \%)$, respectively (Fig. 7c). At none of the centers was an association between the occurrence of UBUs and injected activity found (center $\mathrm{A}: \mathrm{U}=1649.00, \mathrm{Z}=0.95$, $p=0.34$; center $\mathrm{B}: \mathrm{U}=1806.50, \mathrm{Z}=-0.47, p=0.64$; center $\mathrm{C}: \mathrm{U}=446.50, \mathrm{Z}=1.78, p=0.08$; center $\mathrm{D}: \mathrm{U}=17.00$, $\mathrm{Z}=-1.95, p=0.05$ ) (Supp. 4). The mean and SD for injected activity at center A was $233 \pm 40.4 \mathrm{MBq}$, for center B was $299.4 \pm 35.2 \mathrm{MBq}$, for center $\mathrm{C}$ was $282 \pm 34.9 \mathrm{MBq}$,

$(p=.05)$. c Bar charts with the percentage distribution of the frequency of UBUs for each tumor size. d Bar charts with the percentage distribution of the frequency of UBUs for each ISUP score

and for center $\mathrm{D}$ was $258.8 \pm 50.0 \mathrm{MBq}$ (Supp. 5). There was also no association between the occurrence of UBUs and injected activity in digital PET/CT $(\mathrm{U}=341.50, \mathrm{Z}=0.45$, $p=0.66)$ and digital PET/MRI $(\mathrm{U}=283.50, \mathrm{Z}=0.99$, $p=0.32)($ Supp. 6).

\section{Discussion}

Our study showed a high frequency $(51.4 \%)$ of UBUs in patients undergoing $\left[{ }^{18} \mathrm{~F}\right]-\mathrm{PSMA}-1007 \mathrm{PET}$, and this number is higher compared to previously reported unspecific findings on [ ${ }^{68} \mathrm{Ga}$ ]-PSMA-11 [21]. The first publication that reported an increased number of benign and unspecific lesions using $\left[{ }^{18} \mathrm{~F}\right]$-PSMA-1007, compared to $\left[{ }^{68} \mathrm{Ga}\right]-$ PSMA-11, also found a substantially higher number of unclear or likely benign findings in the bones $(48 \%$ vs. 
Fig. 5 The left part of the Sankey diagram shows the frequencies of patients with UBU-related clinical problems (CP) or without UBU-related CPs for early biochemical recurrence (BCR), tumor evaluation (TE), and tumor staging. The right part of the Sankey diagram shows the percentages of patients with and without follow-up examinations

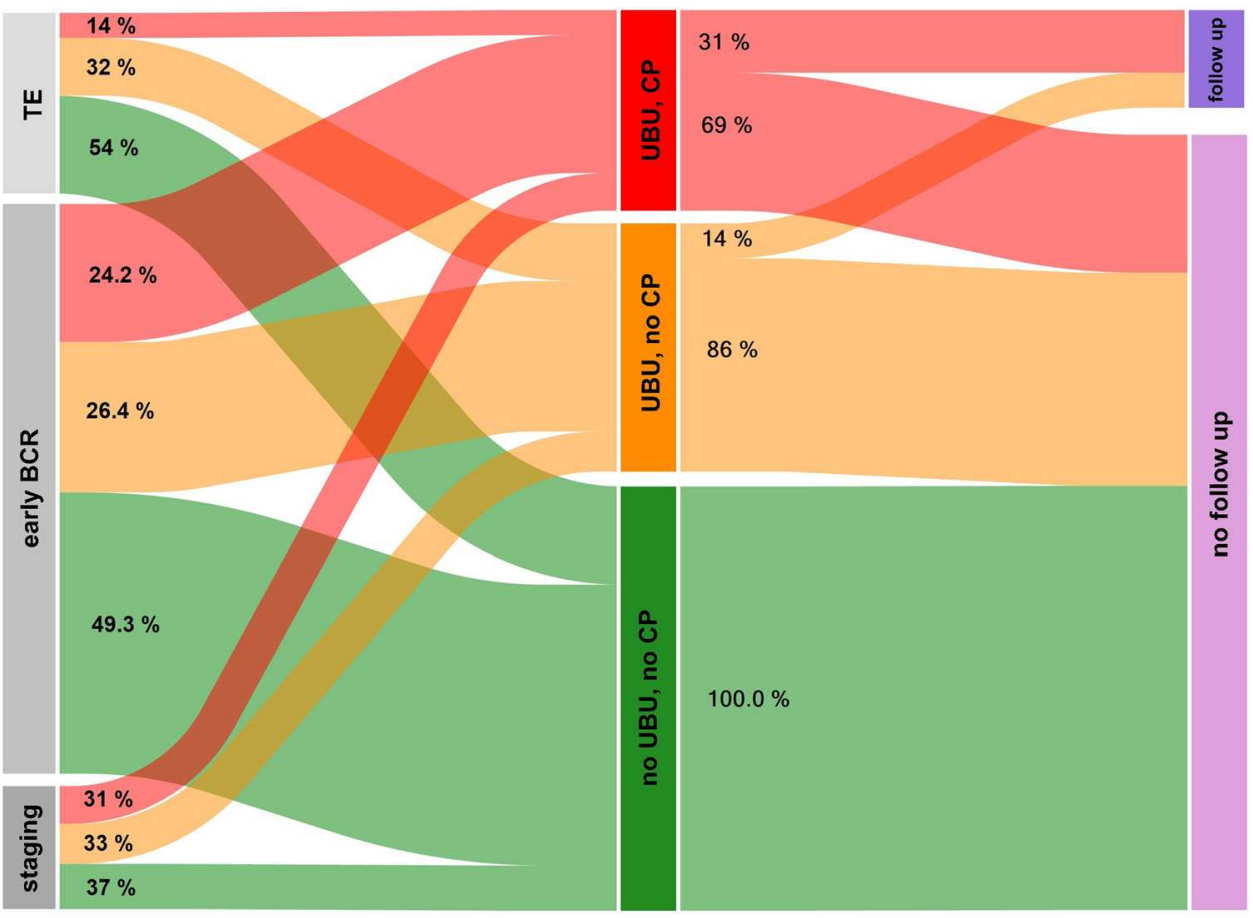

UBU present and clinical problem (CP)

UBU present and no clinical problem (no CP)

UBU absent, no clinical Problem (no CP)
$14.7 \%$ ), lymph nodes (39.2\% vs. $13.7 \%)$, and ganglia (66.7\% vs. $11.8 \%$ ) [22]. The lower positron energy with higher spatial resolution and the higher signal-to-background ratio due to the longer half-life of $\left[{ }^{18} \mathrm{~F}\right]$ compared to $\left[{ }^{68} \mathrm{Ga}\right]$ were suggested as possible explanations for this higher incidence [22]. Immunohistochemistry studies have shown that PSMA is not only expressed in prostate tissue, but is also present in inflammatory and neovascular tissue [25, 26]. Activated granulocytes in the bone marrow might therefore also lead

Table 2 Outcome for biopsy and post-radiotherapy PSA

\begin{tabular}{lll}
\hline & SUV $_{\max }$ & $\begin{array}{l}\text { Outcome biopsy and post- } \\
\text { radiotherapy PSA }\end{array}$ \\
\hline Biopsy & & \\
Pelvis & 3.3 & Hyperplastic bone marrow \\
Pelvis & 9.5 & Paget's disease \\
Pelvis & 9.9 & Hyperplastic bone marrow \\
Post-radiotherapy PSA & \\
Rib & 2.5 & Malignant \\
Rib & 6.8 & Benign \\
Spine & 5.7 & Malignant \\
\hline
\end{tabular}

Post-radiotherapy outcome was defined as benign, if PSA level raised after radiation of the UBU. Post-radiotherapy outcome was defined as malignant, if PSA level significantly dropped after radiation of the UBU to focal bone marrow uptake; therefore, bone marrow islands especially in rips and extremities might be a reason for focal uptake. Furthermore, other bone changes such as fibrous dysplasia or Paget's disease have been suggested as reason for focal bone uptake [27, 28].

Ultimately, the exact mechanism remains unclear; given that UBUs usually persist in follow-up scans, a morphological correlate seems likely. In our cohort, only three UBUs were biopsied, all of them localized in the pelvis and all diagnosed as benign (two hyperplastic bone marrow and one Paget's disease).

In our cohort, there was no association between UBU and age, ISUP score, tumor size (T classification), or PSA level, further underlining the difficulty of interpreting the lesions. The lack of correlation between clinical parameters for tumor aggressiveness with UBU presence further supports the hypothesis that these findings are not cancer related. The most common site of UBU was in the ribs, followed by the pelvis and spine. Wang et al. analyzed the distribution of prostate cancer bone metastases based on bone scans [29] and found that patients with a low number of lesions were most likely to have metastases in the spine, followed by the pelvic bones. They also found that only $1 \%$ of patients had bone metastasis outside the spine and pelvis without also having metastasis in those regions. This reinforces the theory that singular or multiple UBUs in the ribs 
Fig. 6 The Sankey diagram shows the percentages of UBUs that were followed up by imaging, biopsy, and post-radiotherapy PSA, as well as the final assessment of the lesions after follow-up (malignant, benign, and unknown)

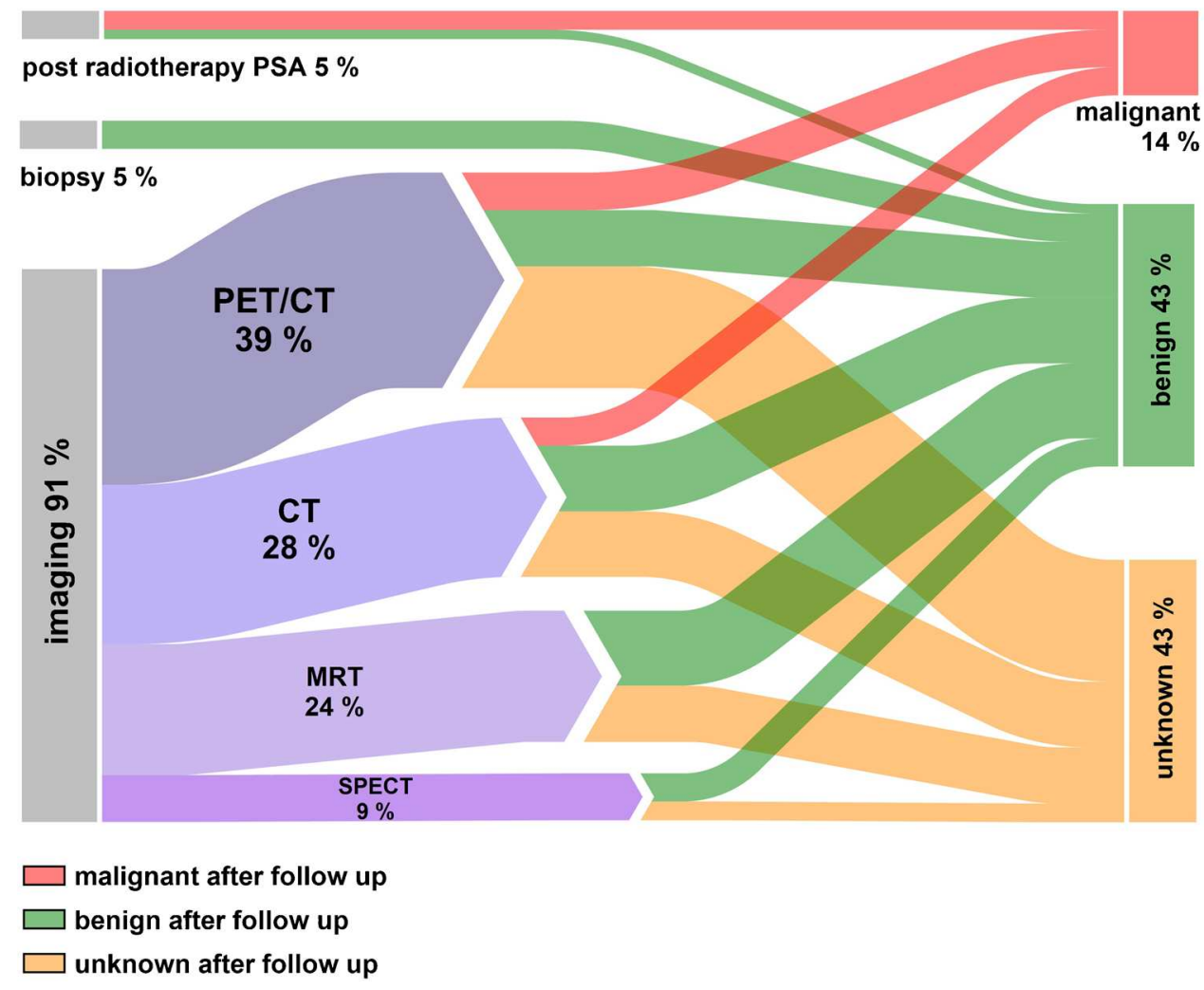

without coexisting suspicious lesions in the spine or pelvis are most likely benign. However, although unlikely, there are cases with solitary bone metastasis in the sternum or ribs, as shown in Fig. 2a-c. More sensitive imaging tools than bone scans might also detect more uncommon locations of bone lesions.

Bone metastasis occurs in approximately $10 \%$ of patients with newly diagnosed prostate cancer, rising to $80-90 \%$ of patients in the advanced stage [30-32]. Therefore, accurate assessment of bone involvement has a direct impact on therapy at every stage in the course of the disease; especially in patients undergoing staging or scanning for early BCR, an M1b situation completely shifts the therapeutic approach from curative to palliative [15].

There was a wide variation of frequencies of UBUs between the different centers, ranging from 36 to $69.7 \%$. This difference might be attributed to the higher scanner sensitivity, given that center A, at $69.7 \%$, was the only institution with digital PET technology. Several studies have shown improvements in lesion detection with digital
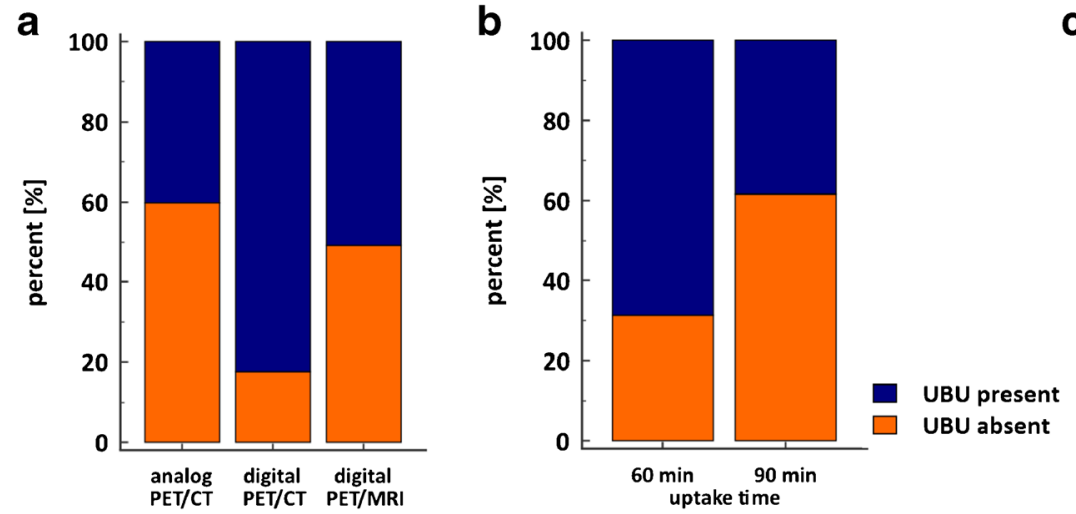

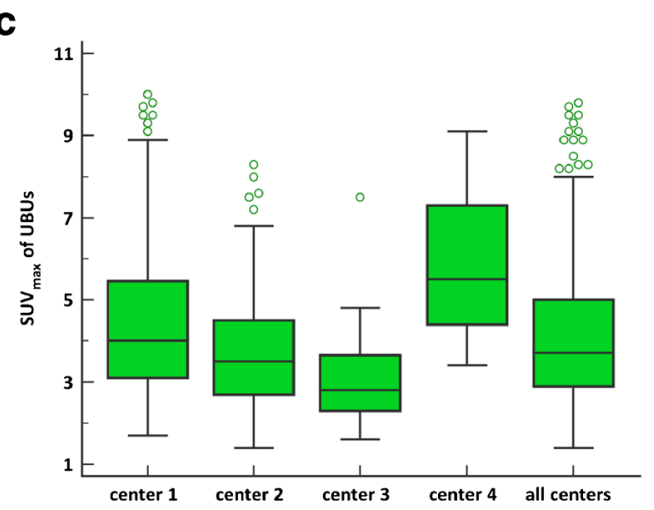

Fig. 7 a Bar charts with the percentage distribution of the frequency of UBUs for analog PET/CT scanners, digital PET/CT scanners, and digital PET/MRI scanners. b Bar charts with the percentage distribu- tion of the frequency of UBUs for PSMA uptake time. $\mathbf{c}$ Box-whisker plots showing the $\mathrm{SUV}_{\max }$ for UBUs at each center separately and for all centers combined 
scanners compared to analog systems for ${ }^{18}$ F-FDG PET, possibly also leading to higher detectability of UBUs using $\left[{ }^{18}\right.$ F]-PSMA-1007 [33, 34]. This was suggested by Alberts et al., who reported a higher detection rate of both prostate cancer lesions and benign PSMA-avid lesions with digital than with analog systems [35]. When comparing digital PET/MR with analog PET/CT, this difference was not visible, and this could be due to the slightly reduced sensitivity of digital PET detectors in MRI scanners due to coils and the magnetic field [36]. It can therefore be assumed that, with more installations of high-end digital PET systems, the frequency of UBU in ${ }^{18}$ F-PSMA-1007 imaging will become even more challenging. We also observed a higher incidence of UBU on scan after 90 min compared to 60-min uptake time, and this could be due to an increased visibility of lesions after 90 min due to reduced background uptake.

The increase in UBU in $\left[{ }^{18} \mathrm{~F}\right]$-PSMA-1007 imaging might impair clinical decisions and could lead to an increase in follow-ups with imaging in cases of ambiguity. Due to the significantly higher number of UBUs with $\left[{ }^{18} \mathrm{~F}\right]-\mathrm{PSMA}-1007$, the previously suggested standardized image interpretation for $\left[{ }^{68} \mathrm{Ga}\right]-\mathrm{PSMA}-\mathrm{PET} / \mathrm{CT}$ published by Fanti et al. should not be adopted for $\left[{ }^{18} \mathrm{~F}\right]$-PSMA-1007
[37], or else the frequency of findings defined as pathological could be significantly higher, leading directly to an overdiagnosis of bone metastasis. Figure 8 shows an example of PSMA-avid bone lesions over-diagnosed as bone metastasis in $\left[{ }^{18} \mathrm{~F}\right]$-PSMA-1007 PET/MRI, with a potential impact on the patient's primary treatment protocol.

The clinical significance of lesions is very subjective and can only be evaluated by incorporating clinical information, as well as the patient's decision, which was beyond the scope of this retrospective study. We therefore evaluated the hypothetical impact of UBUs on patient management in the different imaging settings and tried to reflect how often UBUs actually resulted in a CP. Based on this assessment, 80 out of 179 patients with UBUs were considered to have CPs (44.7\%), but despite this relatively high number, only 39 of the $179(21.8 \%)$ had a follow-up examination. This might be partly due the high number of still-unclear results after follow-up imaging (Fig. 5).

This is a retrospective analysis with associated limitations, most important being the lack of histopathological findings for the bone lesions. Histopathological analysis of UBUs was performed in only three out of 179 patients, showing the difficulty of biopsies for lesions without
Fig. 8 Pretherapeutic $\left[{ }^{18}\right.$ F]-PSMA-1007-PET/ MRI staging examination of a 71-year-old patient with prostate cancer, tumor stage pT3b pNO, ISUP 4, and initial PSA of $24.4 \mu \mathrm{g} / \mathrm{l}$. a A whole-body maximum intensity projection showing the PSMA-positive prostate cancer (thin arrow), no lymph node metastasis, an unspecific bone uptake $\left(\mathrm{SUV}_{\max } 7.0\right)$ in one left rib (bold arrow), and a stronger PSMA-positive bone lesion in the right pelvis $\left(\mathrm{SUV}_{\max } 8.7\right)$. b The pelvic bone lesion in PET (arrow head). c A hypointense morphological correlate on T1-weighted imaging. d The lesion on fused PET/MRI (arrow head). After radical prostatectomy, the PSA normalized (several follow-ups with a PSA of $<0.05 \mu \mathrm{g} / \mathrm{l}$ ) without further therapy, suggesting that both bone lesions were most likely benign
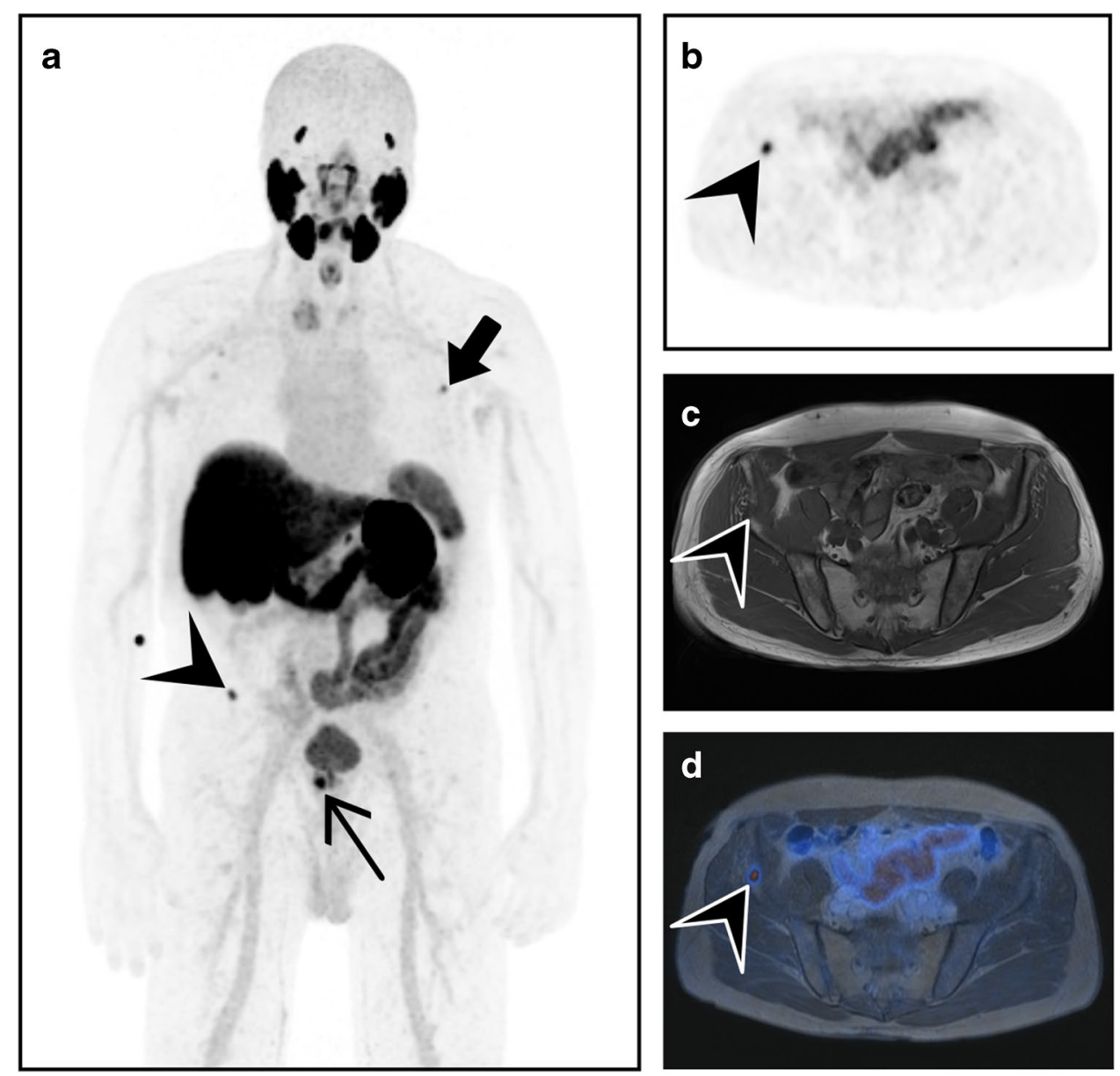
morphological correlates. Imaging or clinical follow-up after radiotherapy was available only in 36 of 179 scans; therefore, $79 \%$ of scans with UBUs did not have follow-up exams, furthermore, while the differences in scanners and protocols at the different centers offer some insights into, for example, the impact of scanner technology on the incidence of UBUs, the asymmetric contributions of the different institutions may have led to a certain bias (e.g., center D having a relatively high number of UBUs (61\%) despite analog detectors, but an overall low number of scans). In addition, unspecific bone lesions were selected and clinical implications interpreted subjectively, without a second reading or consensus.

\section{Conclusion}

Despite the abovementioned advantages of $\left[{ }^{18} \mathrm{~F}\right]$-PSMA-1007 over [ $\left.{ }^{68} \mathrm{Ga}\right]-\mathrm{PSMA}-11$, we found a high incidence of UBUswhich are clinically challenging-in a significant number of patients. If examinations are performed using digital PET scanners, UBUs are detected more frequently than using analog PET scanners. UBUs should be interpreted carefully to avoid over-staging.

Supplementary Information The online version contains supplementary material available at https://doi.org/10.1007/s00259-021-05424-x.

Author contribution Joachim Müller, Klaus Strobel, and Irene. A. Burger contributed to the study conception and design. All authors contributed to the data collection. Alexander Maurer performed the data processing and analysis. The first draft of the manuscript was written by Hannes Grünig, Alexander Maurer, and Irene A. Burger. All authors read and approved the final manuscript.

Funding Open Access funding provided by Universität Zürich. The authors received financial support from the Huggenberger and Jimmy Wirth Foundations.

Data availability The analyzed data may be available from the corresponding author upon reasonable request and with the permission of University Hospital Zurich, University of Zurich, Switzerland.

Code availability Not applicable.

\section{Declarations}

Ethics approval General consent was present or was waived by the local ethics committee.

\section{Consent to participate Not applicable.}

Consent for publication Not applicable.

Competing interests Irene. A. Burger has received research grants and speaker honoraria from GE Healthcare, research grants from Swiss
Life, and speaker honoraria from Bayer Health Care and Astellas Pharma AG.

Open Access This article is licensed under a Creative Commons Attribution 4.0 International License, which permits use, sharing, adaptation, distribution and reproduction in any medium or format, as long as you give appropriate credit to the original author(s) and the source, provide a link to the Creative Commons licence, and indicate if changes were made. The images or other third party material in this article are included in the article's Creative Commons licence, unless indicated otherwise in a credit line to the material. If material is not included in the article's Creative Commons licence and your intended use is not permitted by statutory regulation or exceeds the permitted use, you will need to obtain permission directly from the copyright holder. To view a copy of this licence, visit http://creativecommons.org/licenses/by/4.0/.

\section{References}

1. Wondergem M, van der Zant FM, Broos WAM, Knol RJJ. Clinical impact of PSMA PET in biochemically recurrent prostate cancer; a review of the literature. Tijdschr Urol. 2020;10:109-21. https:// doi.org/10.1007/s13629-020-00296-6.

2. Petersen LJ, Zacho HD. PSMA PET for primary lymph node staging of intermediate and high-risk prostate cancer: an expedited systematic review. Cancer Imaging. 2020;20:10. https://doi.org/ 10.1186/s40644-020-0290-9.

3. Hofman MS, Lawrentschuk N, Francis RJ, Tang C, Vela I, Thomas $\mathrm{P}$, et al. Prostate-specific membrane antigen PET-CT in patients with high-risk prostate cancer before curative-intent surgery or radiotherapy (proPSMA): a prospective, randomised, multicentre study. Lancet. 2020;395:1208-16. https://doi.org/10.1016/S01406736(20)30314-7.

4. Perera M, Papa N, Christidis D, Wetherell D, Hofman MS, Murphy DG, et al. Sensitivity, specificity, and predictors of positive (68)Ga-prostate-specific membrane antigen positron emission tomography in advanced prostate cancer: a systematic review and meta-analysis. Eur Urol. 2016;70:926-37. https://doi.org/10. 1016/j.eururo.2016.06.021.

5. Fendler WP, Calais J, Eiber M, Flavell RR, Mishoe A, Feng FY, et al. Assessment of 68Ga-PSMA-11 PET accuracy in localizing recurrent prostate cancer: a prospective single-arm clinical trial. JAMA Oncol. 2019;5:856-63. https://doi.org/10.1001/jamaoncol. 2019.0096.

6. Janssen JC, Meissner S, Woythal N, Prasad V, Brenner W, Diederichs $\mathrm{G}$, et al. Comparison of hybrid (68)Ga-PSMA-PET/CT and (99m)Tc-DPD-SPECT/CT for the detection of bone metastases in prostate cancer patients: additional value of morphologic information from low dose CT. Eur Radiol. 2018;28:610-9. https://doi. org/10.1007/s00330-017-4994-6.

7. Treglia G, Annunziata S, Pizzuto DA, Giovanella L, Prior JO, Ceriani L. Detection rate of (18)F-labeled PSMA PET/CT in biochemical recurrent prostate cancer: a systematic review and a meta-analysis. Cancers (Basel). 2019;11:1-14. https://doi.org/10. 3390/cancers 11050710.

8. Fendler WP, Ferdinandus J, Czernin J, Eiber M, Flavell RR, Behr SC, et al. Impact of 68Ga-PSMA-11 PET on the management of recurrent prostate cancer in a prospective single-arm clinical trial. J Nuclear Med. 2020;61:1793-9. https://doi.org/10.2967/jnumed. 120.242180 .

9. Ferraro DA, Garcia Schuler HI, Muehlematter UJ, Eberli D, Muller J, Muller A, et al. Impact of (68)Ga-PSMA-11 PET staging on clinical decision-making in patients with intermediate or high-risk 
prostate cancer. Eur J Nucl Med Mol Imaging. 2020;47:652-64. https://doi.org/10.1007/s00259-019-04568-1.

10. Donswijk ML, van Leeuwen PJ, Vegt E, Cheung Z, Heijmink S, van der Poel HG, et al. Clinical impact of PSMA $\mathrm{PET} / \mathrm{CT}$ in primary prostate cancer compared to conventional nodal and distant staging: a retrospective single center study. BMC Cancer. 2020;20:723. https://doi.org/10.1186/ s12885-020-07192-7.

11. Corfield J, Perera M, Bolton D, Lawrentschuk N. (68)Ga-prostate specific membrane antigen (PSMA) positron emission tomography (PET) for primary staging of high-risk prostate cancer: a systematic review. World J Urol. 2018;36:519-27. https://doi.org/10. 1007/s00345-018-2182-1.

12. Yaxley JW, Raveenthiran S, Nouhaud FX, Samaratunga H, Yaxley WJ, Coughlin G, et al. Risk of metastatic disease on (68) galliumprostate-specific membrane antigen positron emission tomography/computed tomography scan for primary staging of 1253 men at the diagnosis of prostate cancer. BJU Int. 2019;124:401-7. https://doi.org/10.1111/bju.14828.

13. van Leeuwen PJ, Emmett L, Ho B, Delprado W, Ting F, Nguyen $\mathrm{Q}$, et al. Prospective evaluation of 68Gallium-prostate-specific membrane antigen positron emission tomography/computed tomography for preoperative lymph node staging in prostate cancer. BJU Int. 2017;119:209-15. https://doi.org/10.1111/bju. 13540.

14. Maurer T, Gschwend JE, Rauscher I, Souvatzoglou M, Haller B, Weirich G, et al. Diagnostic efficacy of (68)gallium-PSMA positron emission tomography compared to conventional imaging for lymph node staging of 130 consecutive patients with intermediate to high risk prostate cancer. J Urol. 2016;195:1436-43. https://doi. org/10.1016/j.juro.2015.12.025.

15. Muller J, Ferraro DA, Muehlematter UJ, Garcia Schuler HI, Kedzia S, Eberli D, et al. Clinical impact of (68)Ga-PSMA-11 PET on patient management and outcome, including all patients referred for an increase in PSA level during the first year after its clinical introduction. Eur J Nucl Med Mol Imaging. 2019;46:889-900. https://doi.org/10.1007/s00259-018-4203-0.

16. The FDA. approves PSMA-targeted drug for PET imaging in men with prostate cancer. BJU Int. 2021;127:267-8. https://doi.org/10. 1111/bju.15361.

17. Dietlein M, Kobe C, Kuhnert G, Stockter S, Fischer T, Schomacker K, et al. Comparison of [(18)F]DCFPyL and [(68) Ga]Ga-PSMA-HBED-CC for PSMA-PET imaging in patients with relapsed prostate cancer. Mol Imaging Biol. 2015;17:575-84. https://doi.org/10.1007/s11307-015-0866-0.

18. Kroenke M, Mirzoyan L, Horn T, Peeken JC, Wurzer A, Wester HJ, et al. Matched-pair comparison of (68)Ga-PSMA-11 and (18) F-rhPSMA-7 PET/CT in patients with primary and biochemical recurrence of prostate cancer: frequency of non-tumor related uptake and tumor positivity. J Nucl Med. 2020. https://doi.org/ 10.2967/jnumed.120.251447.

19. Caribe P, Koole M, D'Asseler Y, Deller TW, Van Laere K, Vandenberghe S. NEMA NU 2-2007 performance characteristics of GE Signa integrated PET/MR for different PET isotopes. EJNMMI Phys. 2019;6:11. https://doi.org/10.1186/ s40658-019-0247-x.

20. Giesel FL, Hadaschik B, Cardinale J, Radtke J, Vinsensia M, Lehnert W, et al. F-18 labelled PSMA-1007: biodistribution, radiation dosimetry and histopathological validation of tumor lesions in prostate cancer patients. Eur J Nucl Med Mol Imaging. 2017;44:678-88. https://doi.org/10.1007/ s00259-016-3573-4.
21. Pernthaler B, Kulnik R, Gstettner C, Salamon S, Aigner RM, Kvaternik H. A prospective head-to-head comparison of $18 \mathrm{~F}-$ fluciclovine with $68 \mathrm{Ga}-\mathrm{PSMA}-11$ in biochemical recurrence of prostate cancer in PET/CT. Clin Nucl Med. 2019;44:e566-73. https:// doi.org/10.1097/RLU.0000000000002703.

22. Kroenke M, Mirzoyan L, Horn T, Peeken JC, Wurzer A, Wester $\mathrm{HJ}$, et al. Matched-pair comparison of (68)Ga-PSMA-11 and (18)F-rhPSMA-7 PET/CT in patients with primary and biochemical recurrence of prostate cancer: frequency of nontumor related uptake and tumor positivity. J Nucl Med. 2020:jnumed.120.251447-jnumed.120. https://doi.org/10.2967/ jnumed.120.251447.

23. Egevad L, Delahunt B, Srigley JR, Samaratunga H. International Society of Urological Pathology (ISUP) grading of prostate cancer-an ISUP consensus on contemporary grading. Blackwell Munksgaard; 2016. p. 433-5.

24. Egevad L, Delahunt B, Srigley JR, Samaratunga H. International Society of Urological Pathology (ISUP) grading of prostate cancer - An ISUP consensus on contemporary grading. APMIS. 2016;124:433-5. https://doi.org/10.1111/apm.12533.

25. Silver DA, Pellicer I, Fair WR, Heston WD, Cordon-Cardo C. Prostate-specific membrane antigen expression in normal and malignant human tissues. Clin Cancer Res. 1997;3:81-5.

26. Chang SS, Reuter VE, Heston WD, Bander NH, Grauer LS, Gaudin PB. Five different anti-prostate-specific membrane antigen (PSMA) antibodies confirm PSMA expression in tumor-associated neovasculature. Cancer Res. 1999;59:3192-8.

27. Froehner M, Toma M, Zöphel K, Novotny V, Laniado M, Wirth MP. PSMA-PET/CT-positive Paget disease in a patient with newly diagnosed prostate cancer: imaging and bone biopsy findings. Case Rep Urol. 2017;2017:1-3. https://doi.org/10.1155/2017/ 1654231.

28. Plouznikoff N, Garcia C, Artigas C, Entezari K, Flamen P. Heterogeneity of 68Ga-PSMA PET/CT uptake in fibrous dysplasia. Clin Nucl Med. 2019;44:E593-4. https://doi.org/10.1097/RLU. 0000000000002609.

29. Wang C, Shen Y. Study on the distribution features of bone metastases in prostate cancer. Nucl Med Commun. 2012;33:379-83. https://doi.org/10.1097/MNM.0b013e3283504528.

30. Park SH, Eber MR, Shiozawa Y. Models of prostate cancer bone metastasis. Methods Mol Biol. 2019;1914:295-308. https://doi. org/10.1007/978-1-4939-8997-3_16.

31. Bubendorf L, Schopfer A, Wagner U, Sauter G, Moch H, Willi N, et al. Metastatic patterns of prostate cancer: an autopsy study of 1,589 patients. Hum Pathol. 2000;31:578-83. https://doi.org/10. 1053/hp.2000.6698.

32. Gandaglia G, Karakiewicz PI, Briganti A, Passoni NM, Schiffmann J, Trudeau V, et al. Impact of the site of metastases on survival in patients with metastatic prostate cancer. Eur Urol. 2015;68:325-34. https://doi.org/10.1016/j.eururo.2014.07. 020 .

33. Lopez-Mora DA, Flotats A, Fuentes-Ocampo F, Camacho V, Fernandez A, Ruiz A, et al. Comparison of image quality and lesion detection between digital and analog PET/CT. Eur J Nucl Med Mol Imaging. 2019;46:1383-90. https://doi.org/ 10.1007/s00259-019-4260-z.

34. Nguyen NC, Vercher-Conejero JL, Sattar A, Miller MA, Maniawski PJ, Jordan DW, et al. Image quality and diagnostic performance of a digital PET prototype in patients with oncologic diseases: initial experience and comparison with analog PET. J Nucl Med. 2015;56:1378-85. https://doi.org/10.2967/jnumed.114. 148338 . 
35. Alberts I, Prenosil G, Sachpekidis C, Weitzel T, Shi K, Rominger A, et al. Digital versus analogue PET in [(68)Ga]Ga-PSMA-11 PET/CT for recurrent prostate cancer: a matched-pair comparison. Eur J Nucl Med Mol Imaging. 2020;47:614-23. https://doi.org/ 10.1007/s00259-019-04630-y.

36. Wollenweber SD, Delso G, Deller T, Goldhaber D, Hullner M, Veit-Haibach P. Characterization of the impact to PET quantification and image quality of an anterior array surface coil for PET/MR imaging. MAGMA. 2014;27:149-59. https://doi.org/ 10.1007/s10334-013-0388-1.
37. Fanti S, Minozzi S, Morigi JJ, Giesel F, Ceci F, Uprimny C, et al. Development of standardized image interpretation for $68 \mathrm{Ga}-$ PSMA PET/CT to detect prostate cancer recurrent lesions. Eur J Nucl Med Mol Imaging. 2017;44:1622-35. https://doi.org/10. 1007/s00259-017-3725-1.

Publisher's note Springer Nature remains neutral with regard to jurisdictional claims in published maps and institutional affiliations. 